\title{
Preterm birth among pregnancies conceived by assisted reproduction techniques in Mumbai, Maharashtra, India
}

\author{
Anushree D. Patil ${ }^{1 *}$, Sanjay L. Chauhan ${ }^{1}$, Achhelal R. Pasi ${ }^{1}$, Rahul K. Gajbhiye ${ }^{1}$, \\ Shalini Singh ${ }^{2}$, Charu Sud ${ }^{1}$, Rana Khan ${ }^{1}$, Jatin S. Shah ${ }^{3}$, Duru S. Shah ${ }^{4}$, Ameet S. Patki ${ }^{5}$, \\ Nitin M. Narvekar ${ }^{6}$, Sanjeev S. Khot ${ }^{7}$
}

\author{
${ }^{1}$ Department of Clinical Research, ICMR-National Institute for Research in Reproductive Health, Mumbai, \\ Maharashtra, India \\ ${ }^{2}$ Department of Reproductive and Child Health, Indian Council of Medical Research (ICMR), New Delhi, India \\ ${ }^{3}$ Director, Mumbai Fertility Clinic, Mumbai, Maharashtra, India \\ ${ }^{4}$ Director, Gynaec World Clinic, Mumbai, Maharashtra, India \\ ${ }^{5}$ Consultant, Fertility Associates, Mumbai, Maharashtra, India \\ ${ }^{6}$ Director, Narvekar Infertility and Endoscopy Clinic, Mumbai, Maharashtra, India \\ ${ }^{7}$ Director, Orrchid Fertility Centre, Mumbai, Maharashtra, India
}

Received: 31 January 2018

Accepted: 06 March 2018

\section{*Correspondence:}

Dr. Anushree D. Patil,

E-mail: rajshrinm@yahoo.co.in

Copyright: (C) the author(s), publisher and licensee Medip Academy. This is an open-access article distributed under the terms of the Creative Commons Attribution Non-Commercial License, which permits unrestricted non-commercial use, distribution, and reproduction in any medium, provided the original work is properly cited.

\section{ABSTRACT}

Background: Preterm births are an enormous global problem on families, medical system and economy. The rates of preterm birth are increasing and one of the contributors is growing use of Assisted Reproduction Techniques (ART) leading to multifetal gestations. Some risk factors for preterm birth are specific to women who conceive by ART. Since there is limited data from India, this pilot study was undertaken to assess the magnitude of preterm birth among pregnancies conceived by ART and to study the contributing factors.

Methods: Clinic based descriptive cohort study through eight ART clinics in Mumbai for one year. Data was collected using an in-depth questionnaire on socio-demographic characteristics, medical history, ART details, course and complications during pregnancy, mode of delivery, pregnancy outcome, risk factors related to preterm birth and neonatal outcome. Complete details of 113 participants who completed the study were analyzed.

Results: Study showed high incidence of preterm birth (76.23\%) among women conceived with ART. Multiple gestations were observed in $45.1 \%$. Pregnancy related complications like heterotrophic pregnancy (3\%), pre eclampsia $(15 \%)$ and gestational diabetes $(11 \%)$ were high. Incidence of caesarean section was very high $(98 \%)$. Neonatal outcome was good with $98 \%$ live births and only 2 still births.

Conclusions: Present study highlights that preterm birth, multiple pregnancies, pregnancy related complications like preeclampsia, gestational diabetes and caesarean sections are very high among women conceived by ART. With growing use of ART there is an urgent need to develop a National ART Surveillance system in India like the one in Centre for Disease Control Atlanta to get complete data on the pregnancy course and outcomes of ART conceptions. Efforts to limit the number of embryos transferred should be strengthened to prevent multiple births.

Keywords: Assisted reproduction techniques, ART, Preterm birth 


\section{INTRODUCTION}

The Centre for Disease Control and Prevention (CDC) defines Assisted Reproductive Technique (ART) as any procedure that entails the handling of both eggs and sperm or of embryos for the purpose of establishing a pregnancy. ${ }^{1}$ Patients who undergo ART procedures are more likely to deliver multiple-birth infants than women who conceive naturally. Multiple births are associated with increased risk for mothers and infants (e.g., pregnancy complications, premature delivery, low-birth weight infants, and long-term disability among infants).

Preterm labor is defined as the onset of labor prior to 37 completed weeks of gestation. ${ }^{2}$ Preterm births are an enormous global problem and causes a huge toll emotionally, physically and financially. Trend data shows that rates of preterm birth are increasing. Key factors contributing to this increase include a rise in the number of pregnancies in women over the age of 35 years, the growing use of ART leading to an increase in the number of twin and higher order multiple births; and the rise in the number of late preterm births (defined as between 34 and 36 weeks of gestation). ${ }^{3}$ Preterm birth and neonatal prematurity related complications are the main cause for longer stay in hospital in ART conceived babies. ${ }^{4}$

Other than the known risk factors for preterm birth, there are also certain risk factors specific to women who conceive by ART which may increase the risk of preterm birth in this group. Maternal characteristics, underlying medical conditions associated with sub fertility and infertility, the use of fertility medications to achieve controlled ovarian hyper stimulation, laboratory conditions, differences in obstetrical management practices, increased proportion of multiple gestations and vanishing twins, or a combination of these factors can be responsible for preterm birth in this group. ${ }^{5}$ However, there is lack of published data on preterm births in pregnancies conceived by ART, especially in India.

The objectives of this pilot study were to understand the magnitude of preterm birth, pregnancy and neonatal outcomes among women conceived by ART in Mumbai and also to explore the contributing factors that could be possibly reduced or prevented.

\section{METHODS}

This clinic based prospective cohort study was undertaken in Mumbai after obtaining the approval from Institutional Ethics Committee. Eight ART centers were chosen where most of the ART conceived pregnancies were either delivered at the same centre or nearby maternity hospitals. These ART centers were representative of Mumbai city.

A total of 409 study participants were enrolled for the study. As there was no published literature on prevalence of pre-term birth (PTB) among women conceived by
ART, sample size was calculated by assuming maximum probability of $50 \%$, level of significance of $5 \%$ and relative precision of $10 \%$. Data was collected by using a standard questionnaire developed at our Institute which was pre-tested. Information was collected on sociodemographic characteristics, medical history, ART details, course and complications during pregnancy, mode of delivery, pregnancy outcome and risk factors related to preterm birth.

Women conceived by ART and willing to share the required medical information were included in the study. The patients were recruited after pregnancy was confirmed by ultrasound. Medical Research Officer and Medical Social Worker were appointed on the project to follow up the study participants. The relevant details like maternal characteristics, fertility factors, obstetrical management during pregnancy and pregnancy outcome details of the enrolled participants were obtained from medical records and telephonic conversation with participants. Preterm labour was considered whenever labour occurred before 37 weeks of gestation. Risk factors for preterm birth in this cohort of women conceived by ART were analyzed. Neonatal outcome up to 4 weeks following delivery were available for analysis.

A total of 409 women undergoing ART procedure and with USG confirmation of pregnancy were approached for the study. Out of this 89 women $(21.7 \%)$ went back to their hometown for antenatal care and delivery. Remaining 320 women were given information about the study and invited to participate. Out of this 160 women $(50 \%)$ were unwilling to participate and share their ART details which they felt was very personal information to be shared for research purpose in spite of assurance of confidentiality. One hundred and fifty-two women agreed to take part in the study. Thirty-nine women were loss to follow up. Complete descriptive data of ART details and maternal and neonatal outcomes were analyzed for 113 women, which is presented in the results section.

Data analysis was done by using SPSS version-19 software. Proportion of preterm birth among women conceived through ART and associated risk factors were measured in terms of percentage.

\section{RESULTS}

The descriptive details of the ART details were analysed. The mean age of wife was 33.1 \pm 5.9 SD (years), and husband was $33.5 \pm 6.4 \mathrm{SD}$ (years). The minimum age of wife being 22 years and husband 25 years, while maximum age of wife was 48 years and husband 53 years. Seventy nine percent of women and $85.99 \%$ husbands had completed their graduation. Majority of the women i.e. $67 \%$ were unemployed when they conceived, $30 \%$ were professionals and $3 \%$ were self-employed. Thirty percent of male partners were professionally employed and $44 \%$ had their own business. Most couples (79\%) belonged to the middle-income group, $5 \%$ were in 
the low-income group and $16 \%$ were in high income group.

The mean height of study participants was $157.9 \mathrm{~cm}$, and weight $61.8 \mathrm{~kg}$, giving a mean BMI of $24.5 \mathrm{Kg} / \mathrm{m} 2$. Anaemia was seen in $6.9 \%$ of participants, but all were euthyroid at the time of enrollment since thyroid status was corrected at the time of ART conception. None of the women were smokers or alcoholics.

Mean duration of infertility in the study participants was 7.2 years (minimum being 0.8 years and maximum 20 years). Primary infertility was seen in $81.2 \%$ of couples while $18.8 \%$ had secondary infertility. Female factor was the cause of infertility in $48.8 \%$ of couples, male factor in $29.2 \%$ of couples and combined factor in $22 \%$ of couples. The details of treatment given for ART are listed in Table 1.

Table 1: ART treatment details in the study group.

\begin{tabular}{|lll|}
\hline ART details & Number & Percentage \\
\hline $\begin{array}{l}\text { Conventional in-vitro } \\
\text { fertilization (IVF) }\end{array}$ & 78 & 69 \\
\hline $\begin{array}{l}\text { Intra cytoplasmic sperm } \\
\text { injection (ICSI) }\end{array}$ & 35 & 31 \\
\hline Short protocol & 16 & 11.50 \\
\hline Long protocol & 60 & 52.21 \\
\hline Self-oocyte & 49 & 43.36 \\
\hline Donor oocyte & 64 & 56.64 \\
\hline Husband sperm & 81 & 71.68 \\
\hline Donor sperm & 32 & 28.32 \\
\hline
\end{tabular}

The pregnancy outcomes were also observed and described in the Table 2.

Table 2: Outcome of pregnancy in the study participants $(n=113)$.

\begin{tabular}{|lll|}
\hline Pregnancy outcome details & Number & Percentage \\
\hline First trimester abortions & 4 & 3.53 \\
\hline Second trimester abortions & 5 & 4.42 \\
\hline $\begin{array}{l}\text { Heterotrophic ectopic } \\
\text { pregnancies }\end{array}$ & 3 & 2.65 \\
\hline Congenital anomalies & 1 & 0.88 \\
\hline Still births & 1 & 0.88 \\
\hline Early neonatal deaths & 3 & 2.65 \\
\hline Multiple pregnancies & 51 & 45.1 \\
\hline Singleton pregnancies & 62 & 54.8 \\
\hline Preterm deliveries & 77 & 68.14 \\
\hline Full term deliveries & 24 & 21.23 \\
\hline
\end{tabular}

Multiple gestations were observed in 51 participants $(45.1 \%)$ and singleton pregnancies in 62 participants $(54.9 \%)$. The high incidence of multiple gestations can be correlated with the high incidence of preterm births observed in this study. All women were given progesterone supplementation to support the pregnancy till 12 weeks of pregnancy. 18 women $(15.9 \%)$ required the use of tocolytic agents to arrest or prevent preterm labor in the late second and early third trimester.

Fifty-one women $(45.1 \%)$ had antepartum haemorrhage. Out of these forty-one women (35.9\%) had bleeding episodes during the 1st trimester, of which 4 sustained pregnancy losses. Ten women had bleeding episodes during the 2nd trimester, 5 of which were terminated. Physical factors like incompetent os were observed in 3 out of the 5 mid trimester abortion cases. All women were monitored using ultrasounds during the entire duration of pregnancy.

Table 3 describes the medical complications during pregnancy in the study group.

Table 3: Details of medical complications during pregnancy in study group $(n=113)$.

\begin{tabular}{|lll|}
\hline Pregnancy complication & Number & Percentage \\
\hline Preeclampsia & 17 & 15.04 \\
\hline Oligohydramnios & 6 & 5.3 \\
\hline Gestational diabetes & 12 & 10.6 \\
\hline cervical cerclage & 27 & 23.9 \\
\hline $\begin{array}{l}\text { Premature rupture of } \\
\text { membranes (PROM) }\end{array}$ & 16 & 14.2 \\
\hline Multiple pregnancy & 51 & 45.1 \\
\hline
\end{tabular}

Twenty-four women delivered at term and 77 had preterm deliveries. The incidence of preterm births in this study was observed to be $76.2 \%$. Sixty-two women underwent elective LSCS, 36 were emergency LSCS. Only three women had vaginal deliveries, out of which 1 was a twin pregnancy. The incidence of caesarean section in pregnancies conceived by ART was very high (98\%).

Women were admitted during their pregnancy at various periods of gestations requiring hospital stay varying from 1 day to 65 days. Risk factors associated with preterm birth like age of wife, BMI, haemoglobin levels, duration of infertility, economic condition, multiple pregnancy, history of periodontal disease, urinary tract infection, reproductive tract infection and use of short protocol were analysed as described in table 4 . Risk factor analysis concluded that multiple pregnancy is the risk factor significantly associated with preterm birth among pregnancies conceived by ART.

Sixty-six $(49.6 \%)$ of the babies were males and 73 $(54.8 \%)$ were females. The incidence of live birth was $98 \%$ with just 2 still births, both extremely preterm deliveries. Only one case of congenital anomaly i.e. VACTERL was detected. Sixty-two babies (44.6\%) required NICU stay. All babies were discharged after a short course of stay in NICU. These babies were followed up for one month after delivery and were found to be gaining weight. 


\section{DISCUSSION}

Assisted reproductive techniques and its variants have become routine infertility treatments in industrialized countries. ${ }^{6}$ The incidence of pregnancies by ART (IVF and ICSI) and the incidence of preterm birth associated with pregnancies conceived by ART is increasing over the years globally as well as in India. Infertile women seem to have predisposition to giving preterm birth and low birth weight babies..$^{7-10}$ The precise reasons for this increase in adverse outcomes are not clear, but potential factors include: maternal characteristics, underlying medical conditions associated with sub fertility and infertility, sperm factors, the use of fertility medications to achieve controlled ovarian hyper stimulation, laboratory conditions during embryo culture, culture medium, differences in obstetrical management, increased proportion of multiple gestations and vanishing twins, or a combination of these factors. ${ }^{5}$

Late age of conception and pregnancy as observed in the study predisposes the woman to age related complications during pregnancy and higher incidence of preterm birth and operative delivery. The effects of advanced maternal age also need to be considered since many women who undergo IVF are older and these women are therefore more likely to have pregnancy complications which would end in preterm births.

In this study, the mean duration between diagnosis of infertility and actual conception of a viable fetus was 7.2 years which is a period of stress, both emotional and financial for the couple. Most women were unemployed at the time of conception and on enquiry it was found that they also leave jobs since long fertility treatment causes loss of wages due to absence from work. Most couples seeking treatment with ART were from middle income group which emphasises the need to have low cost ART clinics in public health facilities. Mean BMI being 24.5 in this specific group, this could be an important predisposing factor for pregnancy complications. Since most women received pre-conception care the incidence of anaemia was low and most thyroid disorders were corrected and all women were euthyroid at time of conception. None of the women in our study group were smokers or alcoholics which is an important predisposing factor for preterm birth.

There was no statistical difference between the type of protocol used (long vs short) for ovulation induction and preterm birth $(\mathrm{p}>0.05)$. Since ovulation induction was used in these women, all women received progesterone supplementation in the first trimester to support their pregnancy. In spite of the use of progesterone and other tocolytic agents to arrest or prevent preterm labor, many women had preterm labor.

Donor oocytes were used in more than $50 \%$ cycles while donor sperms were used in one third cases. The gametes used, either self or donor, also did not affect the rate of preterm delivery in our study cohort. In majority of the women fresh gametes and embryos were used. There was also no association with preterm birth whether the women conceived in fresh cycle or a frozen embryo transfer cycle.

Risk factor analysis as shown in Table IV highlights that multiple pregnancies was the main factor which was significantly associated with preterm deliveries $(\mathrm{p}<0.01)$. The incidence of preterm birth in our study cohort was high $(76.23 \%)$ majorly attributed to multiple pregnancies (45.1\%).

Table 4: Risk factors associated with preterm birth.

\begin{tabular}{|llll|}
\hline Risk factors & $\begin{array}{l}\text { Preterm } \\
\text { birth }\end{array}$ & $\begin{array}{l}\text { Full } \\
\text { term } \\
\text { birth }\end{array}$ & $\begin{array}{l}\text { P } \\
\text { value }\end{array}$ \\
\hline $\begin{array}{l}\text { Age of wife } \\
\text { Mean } \pm \text { SD) }\end{array}$ & $33.6 \pm 5.8$ & $32.8 \pm 5.7$ & 0.5 \\
\hline BMI (Mean \pm SD) & $24.1 \pm 3.8$ & $25.1 \pm 3.7$ & 0.2 \\
\hline $\begin{array}{l}\text { Haemoglobin } \\
\text { (Mean } \pm \text { SD) }\end{array}$ & $11.7 \pm 1.2$ & $1.8 \pm 1.1$ & 0.9 \\
\hline $\begin{array}{l}\text { Duration of infertility } \\
\text { (Mean } \pm \text { SD) }\end{array}$ & $7.8 \pm 5.2$ & $25.1 \pm 3.7$ & 0.5 \\
\hline $\begin{array}{l}\text { Annual income in Rs } \\
\text { lakhs (Mean } \pm \text { SD) }\end{array}$ & $24.1 \pm 3.8$ & $7.0 \pm 3.1$ & 0.4 \\
\hline $\begin{array}{l}\text { Multiple pregnancy } \\
\text { H/O periodontal }\end{array}$ & 45 & 6 & 0.01 \\
\hline disease & 0 & 1 & 0.26 \\
\hline H/O UTI & 4 & 2 & 0.5 \\
\hline H/O treatment of RTI & 6 & 1 & 0.6 \\
\hline Use of short protocol & 12 & 4 & 0.17 \\
\hline
\end{tabular}

Danish researchers found that among more than 20,000 women who gave birth at their hospital between 1989 and 2006, those who had conceived through ART had a higher risk of preterm delivery. ${ }^{11}$ A meta-analyses showed that singleton pregnancies resulting from IVF have increased rates of preterm birth compared with naturally conceived pregnancies. ${ }^{12} \mathrm{~A}$ recent large-scale study from United States concluded that even among singleton births to primiparas, those conceived by ART had increased risk for preterm birth. ${ }^{13}$ The characteristics of Indian population is different from other countries in terms of physical, socio cultural and various other characteristics and hence the need to have robust Indian data through an ART surveillance system.

Studies have shown that there was an increase in the pregnancy related complications in women who conceived with ART. The incidence of heterotrophic pregnancy, in a natural conception has been estimated to be 1 in 30000 pregnancies while in our study the incidence was 3 in 100 pregnancies which is similar to the incidence found in other studies among ART conceptions. This high incidence could be due to higher number of embryos transferred. 
In this cohort there was higher incidence of pre-eclampsia $(15.04 \%)$, oligohydroamnios $(5.3 \%)$ and gestational diabetes $(10.6 \%)$. These maybe related to the increased age at conception.

The incidence of caesarean section in our study group was very high (98\%). Obstetricians lean towards caesarean delivery particularly in twin pregnancy. ${ }^{14}$ Their anxiety and concern while managing pregnancies in infertile women may increase the rate of caesarean section in this group of patients, although studies have suggested that the outcomes were not improved by caesarean section. Increased rates of caesarean section and preterm delivery are the main obstetric concerns in pregnancies conceived through ART.

Preterm birth and neonatal prematurity related complications are the main cause for longer stay in hospital in ART conceived twins. ${ }^{4}$ In this study these women were admitted during their pregnancies, at various periods of gestations, requiring hospital stay varying from 1 day to 65 days. The positive aspect of this cohort was that in spite of the high incidence of preterm birth, the incidence of live babies was $98 \%$ and the study showed just 2 still births. The stillbirths were seen in very early preterm deliveries. Though preterm births were high, neonatal care was excellent resulting in a very high take home baby rate.

Limitations of the study small sample size (since this was a pilot study) is a limitation of the study. ART and infertility being a very private aspect of a patient's personal life, a lot of women are unwilling to share the medical details even after being explained about the confidentiality. The refusal rate was much higher than expected which shows that the stigma associated with infertility and its treatment is deep rooted in all strata's of our society.

\section{CONCLUSION}

Present study highlights that preterm birth, multiple pregnancies, pregnancy related complications like preeclampsia, gestational diabetes and caesarean sections are very high in this cohort of women conceived by ART. As a crucial step towards reaching the United Nation's Millennium Development Goals to reduce neonatal mortality and improve maternal health, strategies for identifying and reducing preterm birth is essential.

\section{Recommendations}

With growing use of ART there is a strong need to develop a National ART Surveillance system in India like the one in CDC Atlanta. Mechanisms need to be inbuilt in the reporting systems like National ART Registry of India to get complete data on the pregnancy course and outcomes of ART conceptions. To reduce multifetal pregnancies, efforts to limit the number of embryos transferred in each ART procedure should be continued and strengthened. Epidemiological studies with large sample size after adjusting for confounding factors to understand the underlying causes of preterm birth specifically in this group need to be undertaken in India. Adverse maternal and infant outcomes (e.g., preeclampsia, preterm delivery and low birth weight) and the need for good neonatal intensive care should be emphasized when counseling patients considering ART. The course of pregnancies and the health of children born after ART are two of the most important 'outcome parameters' of the quality of the outcomes. The most appropriate end point after assisted reproduction should also include preterm or term birth as measure of success and this should be included in the National ART Registry of India.

\section{ACKNOWLEDGMENTS}

Authors would like to thank project staff and all present study participants for sharing their medical information.

\section{Funding: Indian Council of Medical Research Conflict of interest: None declared \\ Ethical approval: The study was approved by the Institutional Ethics Committee}

\section{REFERENCES}

1. Reynolds MA, Schieve LA, Martin JA, Jeng G, Macaluso M. Trends in multiple births conceived using assisted reproductive technology in United States 1997-2000. Pediatr. 2003;111:1159-62.

2. Singh U, Singh N, Seth S. A prospective analysis of etiology and outcome of preterm labor. J Obstet Gynaecol India. 2007;57(1):48-52.

3. March of Dimes. White paper on preterm birth, The Global and Regional Toll. Available from: http://marchofdimes.com/files/66423_MODComplete.pdf. Accessed on November 2009.

4. Baxi A, Kaushal M. Outcome of twin pregnancies conceived after assisted reproductive techniques. J Hum Reprod Sci. 2008;1(1):25-8.

5. Paulson R. Pregnancy outcome after assisted reproductive technology. Available from http://www.uptodate.com/contents/pregnancyoutcome-after-assisted-reproductive-technology. Accessed 21 $1^{\text {st }}$ April 2017.

6. Powell K. Fertility treatments: Seeds of doubt. Nature. 2003;422:656-8.

7. Bergh $\mathrm{T}$, Ericson A, Hillensjo $\mathrm{T}$, Nygren $\mathrm{KG}$, Wennerholm UB. Deliveries and children born after in-vitro fertilization in Sweden 1982-95: A retrospective cohort study. Lancet. 1999;354:157985.

8. Sutcliffe AG. Health risks in babies born after assisted reproduction. BMJ. 2002;325:117-8.

9. Kallen B, Olausson PO, Nygren KG. Neonatal outcome in pregnancies from ovarian stimulation. Obstet Gynecol. 2002;100:414-9. 
10. Dhont M, De Sutter P, Ruyssinck G, Martens G, Bekaert A. Perinatal outcome of pregnancies after assisted reproduction: A case-control study. Am J Obstet Gynecol. 1999;181:688-95.

11. Wisborg K, Ingerslev HJ, Henriksen TB. In vitro fertilization and preterm delivery, low birth weight, and admission to the neonatal intensive care unit: a prospective follow-up study. Fertil Steril. 2010;94(6):2102-6.

12. Blickstein I. Does assisted reproduction technology per se increase the risk of preterm birth? BJOG. 2006;113(3):68- 72.

13. Dunietz GL, Holzman C, McKane P, Li C, Boulet SL, Todem D, et al. Assisted reproductive technology and the risk of preterm birth among primiparas. Fertility and sterility. 2015;103(4):974-9. 14. Yang Q, Wen SW, Chen W, Krewshi D, Fung KF, Walker M. Neonatal death and morbidity in vertexnonvertex second twins according to mode of delivery and birth weight. Am J Obstet Gynecol. 2005;192:840-7.

Cite this article as: Patil $\mathrm{AD}$, Chauhan SL, Pasi AR, Gajbhiye RK, Singh S, Sud C, et al. Preterm birth among pregnancies conceived by assisted reproduction techniques in Mumbai, Maharashtra, India. Int J Reprod Contracept Obstet Gynecol 2018;7:1548-53. 